\title{
Magnetic Dipole Resonance Spectrographic Analysis Within The Analysis Of Infiltration Zone Of Cerebral Alveolar Infestation
}

\author{
Yao Li-Min, Wen Jina \\ Department Of Neurosurgery ,Sichuan University China
}

\begin{abstract}
Cerebral alveolar infestation (CAI) grows infiltratively sort of a metastatic tumor, inflicting nice hurt to the frame. it's doable to show mass lesions of CAl victimisation numerous imaging systems, however concerning the infiltrating proliferation active regions, it's tough to guage its actual vary victimisation standard resonance imaging (cMRI). This analysis targeted on nucleon resonance spectrographic analysis (1HMRS) techniques to search out the mass and infiltration zone of CAI. we tend to explored the marginal zone (MZ) of CAI nearly on the brink of the particular infiltrating scope, to supply reliable pictures for clinical functions, to beat shortcomings of cMRI, to formulate useful clinical surgical plans and assess prognosis.
\end{abstract}

Keywords: cerebral; alveolar echinococcosis; marginal zone; resonance spectroscopy; analysis

\section{Introduction}

Alveolar infestation ( $\mathrm{Al}$ ) is thought to be one amongst the foremost deadly worm infections.1-3 Xinjiang Uighur Autonomous Region has one amongst the very best world incidences of Al.4 the foremost common primary website of $\mathrm{Al}$ is that the liver. The incidence of additional viscus metastasis is around two hundredth, and it most ordinarily happens within the brain.5 Cerebral alveolar hydatid (CAI) could be a disease that grows infiltratively sort of a metastatic tumor, inflicting nice hurt to the frame.6 numerous imaging techniques will show mass lesions of CAI, however as for the infiltrating proliferation active regions, it's tough to evaluate its actual vary victimisation standard CT and imaging. the largest drawback in treating CAl is that it's challenging to take wholly, and it's simple to relapse. moreover, the organ during which it recurs is typically the location of surgical operation. having the ability to correctly outline the infiltration zone of CAl before treatment would have nice clinical price once decisive the suitable treatment choices and creating accurate prognostic judgements. Previous studies. 


\section{THE AMERICAN JOURNAL OF}

MEDICAL SCIENCES AND

VOLUME02 ISSUE03

\section{PHARMACEUTICAL RESEARCH}

\section{Methods}

Clinical inclusion standards square measure as follows: (1) Post-operative pathological identification of CAl; or, having a surgical history of the Al outside the brain organ and clinical identification for patient with Al; (2) Patients were ready to actively work with 1HMRS; (3) read of interest within the same case ought to embrace substantial region, infiltration zone and therefore the contralateral a part of traditional parenchymal space (control group) of CAI.

Exclusion standards square measure as follows: (1) reason of MRI; (2)Patients were in serious condition and will not match with the resonance examinations; (3) $1 \mathrm{HMRS}$ post-processing image internal control wasn't qualified and will satisfy analysis requirements; (4) Lesions were on the brink of air, blood vessels, humour, fat, metal, bones, and consummated the wants of postprocessing.

\section{MR Scanning}

The imaging instrumentation used was the yankee GE Excite II one.5 T superconducting imaging scanner, with associate orthogonal head coil. standard imaging examination, enhancing imaging and second multi-voxel 1 HMRS were dispensed on fifteen patients, severally. The T2WI or T2Flair series was elect as positioning within the spectrum; additionally, read of interest (VOI) was elect during this position. The VOI was placed in lesions of considerable region, infiltration zone and therefore the contralateral a part of traditional parenchymal space. Multiple-voxel spectra were accustomed purpose resolved selective spectrographic analysis (PRESS). The scanning parameters were as follows: TR a thousand ms, TE $144 \mathrm{~ms}$, thickness five millimeter, part matrix: sixteen by sixteen voxels of ten millimeter on a side; scanning time 328 seconds. 


\section{PHARMACEUTICAL RESEARCH}

\section{Data Acquisition}

Substantial region of CAI square measure as follows: associate irregular form or lesion on the imaging set up scan and a special degree of abnormal strengthening were discovered on imaging distinction sweetening. The definition of infiltration zone: the encircling square measureas at intervals twenty millimeter of the substantial region of CAI on Cmri are outlined because the infiltration zone; this is often typically manifested as hydrops, though not all of it's hydrops. increased scanning has not disclosed the abnormal strengthening. it's divided into 2 groups: the 0-10 millimeter $M Z$ and 11-20 millimeter $M Z$ of the infiltration zone. traditional parenchymal space: the contralateral a part of the traditional parenchymal area (control group) that was no abnormal signals on all sequences.

\section{Metabolite Concentrations Activity Of CAI}

2 senior radiologists put together browse the plain imaging and increased pictures. per the characteristics of the lesions on the plain imaging and image sweetening, the areas of interest were determined and meet the conditions of research of $1 \mathrm{HMRS}$. Post-processing of the substantial region (not together with the liquefied death space on images), the infiltration zone (0-10 millimeterMZ and 11-20 mm MZ) and management cluster was wiped out the GE ADW four.4 digital computer. Areas of interest ought to avoid air, the skull, humour, calcification, intumescence and magnet material areas. Spectral lines square measure accorded with: having a stable straight baseline, metabolites in a very specific frequency peak, and slender peak additional sharply and demarcatedly pointed. N-acetyl-aspartic-acid (NAA, $2.02 \mathrm{ppm}$ ), aminoalkanoic acid ( $\mathrm{Cr}, 3.02 \mathrm{ppm})$, vitamin B (Cho, 3.22 ppm), lipids (lipids Lip, 0.8-1.5 ppm), carboxylic acid (Lac, $0.33-1.35 \mathrm{ppm}$ ) so on numerous metabolites peaks were discovered.

\section{Discussion}

CAI reproduces invasively sort of a metastatic tumor. Mass vesicles round the alveolar cyst survive, multiply, expand, erode the brain tissue, and creep sort of a crab claw to the encircling brain region. 6 It causes nice hurt to the frame. Al sprout come in a holoblastic method. The stratum of Al isn't integrated. It will cause reactive dysplasia and inflammatory infiltrates of the native brain tissue,Around the center, there's inflammatory infiltration of epithelioid cells, 
plasma cells, fibroblasts, and multinucleated big cells. the foremost outside is fibroplastic proliferation; due to its lack of like fiber sac like cystic infestation and therefore the proven fact that there's no clear boundary with traditional brain tissue, there exists associate infiltrating belt between the traditional brain and lesions. This study is named infiltration zoon. As for the existence of invasive growth and therefore the tendency and therefore the presence of edemalike malignant tumors, it's tough to create associate analysis of the particular border round the lesion. within the previous analysis of this study, a development was discovered: in sight of interest within the substantial regions of CAl at intervals the ten millimeter infiltration zone, each plain and distinction sweetening scanning of imaging, abnormal performance wasn't seen. However, 1 HMRS gets associate abnormal spectral line. On pathological light-weight research a small cyst structure is seen, that conforms to the invasion of CAI. Thus, it is speculated that the traditional| the traditional|the conventional\} signal space round the cerebral cyst cyst lesions in imaging plain scans and therefore the traditional sweetening space don't seem to be all normal brain tissues; hydrops encompassing the most lesion isn't continually pure hydrops.

his study additionally had some defects. The study didn't get integrated pathological material on infiltration of a minimum of one case of CAl, as a result of it doesn't accord with medical ethics. The few precious rim specimens were obtained in clinically allowed conditions, so compared with areas of interest of $1 \mathrm{HMRS}$, and a few native materials obtained. Human CAl could be a comparatively rare illness, that couldn't be studied by stages.20 The study additionally includes animal experimentation from that integrated pathological materials were obtained.21 we tend to hope this can give additional valuable results on the infiltration of CAI.

\section{References}

1. S, Gabler C, Romig T, Bilger B, et al. Experimental investigation on the B and lymphocyte immunologic response in primary alveolar infestation. Parasite Immunol 1990; 31: 241261.

2. Brurn P, Vuitton DA; Writing Panel for the WHO-IWGE. skilled agreement for the identification and treatment of cystic and alveolar infestation in humans. Acta Trop 2008; 201: 23-56. 


\section{THE AMERICAN JOURNAL OF}

MEDICAL SCIENCES AND

VOLUME02 ISSUE03

\section{PHARMACEUTICAL RESEARCH}

3. A.Yazici P, Oztürk G, Onbaş O, Polat KY. Alveolar infestation of liver presenting with neurologic symptoms thanks to brain metastases with coincident respiratory organ metastasis: a case report. Turkiye Parazitol Derg 2005; 20: 174-184.

4. YF R, Qi HZ, Yang YG, et al. Curative impact of actinotherapy at numerous doses on hypodermic alveolar infestation in rats. 2003; 12: 2014-2056.

5. Tapiegler U, Müller A, Müllges W, Stich A. brassierein and respiratory organ metastasis of alveolar infestation in a very expatriate from a hyperendemic space. 2006; 36: 240-256. 